\title{
Safety and efficacy of exercise training in various forms of pulmonary hypertension
}

\author{
Ekkehard Grünig*,++, Mona Lichtblau*,++, Nicola Ehlken*, Hossein A. Ghofrani" \\ Frank Reichenberger ${ }^{\#}$, Gerd Staehler", Michael Halank ${ }^{+}$, Christine Fischer ${ }^{\S}$, \\ Hans-Jürgen Seyfarth ${ }^{f}$, Hans Klose**, Andreas Meyer ${ }^{\# \#}$, Stephan Sorichter ${ }^{\text {"थ }}$, \\ Heinrike Wilkens ${ }^{++}$, Stephan Rosenkranz ${ }^{\S \S}$, Christian Opitz ${ }^{f f}$, Hanno Leuchte ${ }^{\star * *}$, \\ Gabriele Karger $^{\# \# \#}$, Rudolf Speich ${ }^{\top \uparrow \uparrow}$ and Christian Nagel*
}

\begin{abstract}
The objective of this prospective study was to assess safety and efficacy of exercise training in a large cohort of patients with different forms and World Health Organization (WHO) functional classes of chronic pulmonary hypertension (PH).
\end{abstract}

183 patients with $\mathrm{PH}$ (pulmonary arterial hypertension (PAH), chronic thromboembolic $\mathrm{PH}$ and $\mathrm{PH}$ due to respiratory or left heart diseases received exercise training in hospital for 3 weeks and continued at home. Adverse events have been monitored during the in-hospital training programme. Efficacy parameters were evaluated at baseline, and after 3 and 15 weeks.

After 3 and 15 weeks, patients significantly improved the distance walked in 6 min (6MWD) compared to baseline, scores of quality of life, WHO functional class, peak oxygen consumption, oxygen pulse, heart rate and systolic pulmonary artery pressure at rest and maximal workload. The improvement in 6MWD was similar in patients with different PH forms and functional classes. Even in severely affected patients (WHO functional class IV), exercise training was highly effective. Adverse events, such as respiratory infections, syncope or presyncope, occurred in $13 \%$ of patients.

Exercise training in PH is an effective but not a completely harmless add-on therapy, even in severely diseased patients, and should be closely monitored.

\section{KEYWORDS: Exercise, pulmonary hypertension, rehabilitation programme, safety}

D revious studies in rather small cohorts of patients with idiopathic pulmonary arterial hypertension (IPAH) and inoperable chronic thromboembolic pulmonary hypertension (CTEPH) have shown beneficial effects of exercise training as add-on to disease-targeted medical therapy, by increasing exercise capacity [1-3] and quality of life [1]. Exercise training also improved peak oxygen consumption and World Health Organization (WHO) functional class [1], and possibly the clinical outcome, with 1- and 2yr survival rates of 100 and $95 \%$, respectively [4]. Importantly, no severe adverse events occurred in the previously assessed training groups. The improvement in 6-min walk distance (6MWD), reached after 15 weeks' exercise training was unexpectedly high with a mean $\pm \mathrm{SD}$ increase of $96 \pm 61 \mathrm{~m} \mathrm{[1]}$ and of $84 \pm 49 \mathrm{~m}$, respectively [4]. However, training studies cannot be performed in a blinded design and a selection bias towards highly motivated patients cannot be excluded
$[1,4]$. Other studies that applied a less intense outpatient training programme in small cohorts of pulmonary arterial hypertension (PAH) patients did reveal a less pronounced [3], or even no significant $[2,5]$, improvement in $6 \mathrm{MWD}$ or peak oxygen consumption, but an improvement in exercise endurance $[2,5]$ and muscle capillarisation [2]. The effect of training in patients with other aetiologies of pulmonary hypertension $(\mathrm{PH})$, such as associated PAH (APAH) or outof-proportion $\mathrm{PH}$ with left heart failure or respiratory diseases, has not yet been evaluated. Furthermore, it has never been evaluated whether training programmes may be harmful, especially for patients with severe $\mathrm{PH}$ and right heart failure, classified in WHO functional class IV. In these patients with end-stage $\mathrm{PH}$, prognostic relevant effects of medical treatment are usually very limited and it would be of interest if such patients may benefit from a careful, lowdose and closely monitored exercise training as

\section{AFFILIATIONS}

${ }^{*}$ Centre for Pulmonary Hypertension at Thoraxclinic Heidelberg, University of Heidelberg,

${ }^{\S}$ Dept of Human Genetics, University of Heidelberg, \#\#\# Clinic of Rehabilitation Königstuhl, Heidelberg,

\#Dept of Pneumology, University of Giessen, Giessen,

"Clinic Löwenstein, Löwenstein,

+Dept of Pneumology, University of Dresden, Dresden,

${ }^{f}$ Dept of Pneumology, University of Leipzig, Leipzig,

**Dept of Pneumology, University of Hamburg, Hamburg,

\#\# Clinic Mönchengladbach,

Mönchengladbach,

- Dept of Pneumology, University of Freiburg, Freiburg,

${ }^{++}$Dept of Pneumology, University of

Homburg, Homburg,

${ }^{\S \S D e p a r t m e n t ~ o f ~ C a r d i o l o g y, ~}$

University Köln, Köln,

${ }^{f f}$ DRK Clinic Berlin Köpenick, Berlin, and

${ }^{\star * *}$ Clinic Neuwittelsbach, Munich, Germany.

$\because$ Centre for PH, University Hospital,

Zurich, Switzerland.

${ }^{+++}$These authors made an equal

contribution to the study.

CORRESPONDENCE

E. Grünig, Centre for Pulmonary

Hypertension, Thoraxclinic,

University Hospital Heidelberg,

Amalienstrasse 5, D-69126

Heidelberg, Germany

E-mail: ekkehard.gruenig@

thoraxklinik-heidelberg.de

Received:

July 212011

Accepted after revision:

Oct 252011

First published online:

Feb 092012

European Respiratory Journal

Print ISSN 0903-1936

Online ISSN 1399-3003 
well. Safety is an important concern in these severely affected patients, particularly since they would be advised to continue exercise training. $\mathrm{PH}$ patients sometimes experience presyncope and syncope immediately after exercise. The incidence of sudden cardiac death due to exercise is unknown, but probably quite low for mild to moderate exercise. However, it is at least a common clinical experience that heavy exercise can be dangerous in PH. In an animal model, exercise training significantly reduced the survival of those rats that received higher monocrotaline dosage representing a progressive form of $\mathrm{PH}$ [6]. Thus, exercise training in $\mathrm{PH}$ may possibly be both a useful and effective additive therapy to disease-targeted medication in some patients, and a dangerous procedure with severe side-effects, including the risk of syncope or even sudden cardiac death, in others. Therefore, the aim of this study was to prospectively assess the effects of exercise training in a large cohort of patients with severe chronic $\mathrm{PH}$ of different aetiologies and functional classes, including endstage disease with $\mathrm{WHO}$ functional class IV. We wanted to monitor the rate of adverse events during the 3-week inhospital exercise training and to identify patients at risk for adverse events and/or for a non-response to training.

\section{METHODS}

\section{Study population and design}

This prospective study investigated patients with severe chronic $\mathrm{PH}$ who received exercise and respiratory training as add-on to disease-targeted medication between January 2005 and October 2010. The study patients belong to a new cohort, details of which have not been published before. Further inclusion criteria were age 18-80 yrs and WHO functional class II-IV [7]. Patients had to be stable and compensated with optimised medical therapy (such as endothelin antagonists, inhaled or parenteral prostanoids, sildenafil, anticoagulants, diuretics and supplemental oxygen) for at least 2 months before entering the study. Six patients who were newly diagnosed with $\mathrm{PH}$ had an interval of 2-6 months between initiation of a new PH-targeted medical treatment and the start of exercise training, all other newly diagnosed patients had an interval of $>6$ months. All patients in WHO functional class IV had been under maximal medical PHtargeted therapy and were stable even though they were at an unsatisfactory clinical stage.

The diagnosis was established at the participating $\mathrm{PH}$ centres according to current guidelines [8]. All patients underwent a detailed clinical work-up, including right heart catheterisation. Left heart catheterisation and/or computed tomography of the lungs were performed in all patients with $\mathrm{PH}$ due to left heart or respiratory diseases and when clinically indicated. All patients gave written informed consent for this study, which was approved by the ethics committee of the University of Heidelberg (Heidelberg, Germany).

\section{Outcome measures}

Efficacy parameters were prospectively evaluated at baseline, week 3 and week 15, as described previously [1, 4]. 6MWD was carried out under standardised conditions [9]. Health-related quality of life assessment was performed by the Short Form Health Survey (SF-36) [10]. The completed SF-36 questionnaire at baseline was compared to the results after 15 weeks. Assessment of 6MWD, SF-36, and of other efficacy parameters were performed by investigators who were blinded to the clinical data. Changes in WHO functional class, Borg dyspnoea index (with 6 representing no exertion and 20 maximal exertion) [11], and gas exchange were also analysed [12]. Cardiopulmonary exercise testing and stress Doppler echocardiography were carried out during supine bicycle exercise without oxygen supply as described previously [1]. Systolic pulmonary artery pressure (PASP), systolic and diastolic systemic blood pressures, workload, heart rate, ventilation, oxygen uptake $\left(V^{\prime} \mathrm{O}_{2}\right)$, oxygen pulse $\left(V^{\prime} \mathrm{O}_{2} /\right.$ heart rate), and carbon dioxide output were measured continuously. The anaerobic threshold was detected with the V-slope method [12].

\section{Exercise training programme}

The exercise and respiratory training was performed as described previously [1,4] in the Rehabilitation Clinic Königstuhl in Heidelberg. We performed a programme especially developed for $\mathrm{PH}$ patients, with $\geqslant 1.5 \mathrm{~h}$ per day exercise training (in intervals distributed over the day) consisting of interval bicycle ergometer training at low workloads $(10-60 \mathrm{~W})$ at 7 days a week, walking, dumb-bell training of single muscle groups using low weights $(500-1,000 \mathrm{~g})$ and respiratory training at 5 days per week. Maximum heart rate during the training corresponded to $60-80 \%$ of the heart rate reached during cardiopulmonary exercise testing. Oxygen saturation had to be kept above $85 \%$. The training was continued with $\geqslant 30 \mathrm{~min}$ per day at 5 days a week at home for the following 15 weeks.

Beside the physical training, patients received mental training to improve their perception of individual physical abilities and limits. Psychological support was offered to all participants. The training programme was closely supervised by physical therapists; physicians specialised in rehabilitation medicine and $\mathrm{PH}$ experts, as described previously [1]. Adverse events were recorded whenever they occurred. Oxygen saturation and heart rate were monitored continuously throughout the training and used to adjust the training intensity. When patients' oxygen saturation fell below $90 \%$ during exercise they received supplemental oxygen $\left(3-10 \mathrm{~L} \cdot \mathrm{min}^{-1}\right)$ throughout the training. 80 patients who were on long-term oxygen therapy (for 16-24 h per day) before inclusion in this study remained on oxygen throughout the training programme. At discharge from the hospital after 3 weeks, patients received an individualised training manual and ordered a bicycle ergometer for use at home.

\section{Statistical methods}

The analyses were performed by a statistician (C. Fischer). Data are given as mean $\pm \mathrm{SD}$. The intra-group comparisons of baseline and weeks 3 and 15 for 6MWD, workload, Borg dyspnoea index, parameters of gas exchange, PASP, systemic blood pressure and heart rate were conducted by paired t-tests, and the McNemar test for WHO functional class. Summation and subscores of the SF-36 questionnaire were compared by ANOVA, two-tailed t-test, and the Kruskall-Wallis test was used for the comparison of the per cent changes of the 6MWD among WHO functional class. All tests were two-sided and p-values $<0.05$ were considered statistically significant. Bonferroni adjustment for multiple comparisons was performed for comparisons of the primary end-points 6MWD and quality of life parameters. All analyses were carried out with IBM SPSS V19 (IBM Corp., Armonk, NY, USA). 
Efficacy parameters were available for statistical analysis at baseline and after 3 weeks in all patients. Since no adverse events or deaths occurred between week 3 and week 15, we used the last observation (after 3 weeks) carried forward technique as a substitution rule for dropouts. To analyse the characteristics of dropouts and the consequences for the results, we compared the baseline and week 3 data of patients who did not come to the 15 -week visit with those of the patients who completed the study.

\section{RESULTS \\ Study population}

We included 194 patients in the study; 11 (5.6\%) had to be excluded and did not start the rehabilitation programme due to the following reasons. In one patient walking distance was mainly impaired due to peripheral artery occlusive disease, he was referred to further treatment; two patients discontinued due to familial problems; one patient was not included due to severe methicillin-resistant Staphylococcus aureus infection; one patient could not participate due to an acute peroneal palsy after a fall; six patients had to be excluded before entering the exercise training programme because of a clinical instable course and the necessity of additional PH-targeted medication at baseline. Thus, the final study group consisted of 183 patients: 87 patients $(47.6 \%)$ with IPAH or heritable PAH, 46 patients (25.1\%) with APAH, 10.4\% with "out-of-proportion" $\mathrm{PH}$ due to left heart or lung diseases and 31 patients $(16.9 \%)$ with inoperable CTEPH (table 1). Out of proportion PH in left heart disease and lung disease were defined due to current guidelines $[8,13]$.

The APAH subgroup consisted mainly of 19 patients with connective tissue disease (CTD-APAH; 41\%) and 15 patients with congenital heart defect (CHD-APAH; 33\%) (table 1). The patients had been referred from 12 centres. Demographic data, diagnosis, functional class, 6MWD and haemodynamic values are summarised in table 1 . At baseline, most patients $(74.9 \%)$ were in WHO class III, 18 patients $(9.8 \%)$ were classified in $\mathrm{WHO}$ functional class IV. Combination therapies, including two to four PH-targeted agents, were used in $50.8 \%$ of patients (table 2).

\section{Assessment of training effects}

Compared to baseline, mean \pm SD $6 \mathrm{MWD}$ increased significantly after $3(68 \pm 46 \mathrm{~m} ; \mathrm{p}<0.001)$ and 15 weeks $(78 \pm 49.5 \mathrm{~m}$; $\mathrm{p}<0.001$ ) (fig. 1 and table 3 ). The improvement in 6MWD was similar in the patients with IPAH/hereditary pulmonary arterial hypertension (HPAH), APAH, inoperable CTEPH or out-of proportion $\mathrm{PH}$ in left heart or lung diseases (fig. 2). Patients with APAH had a tendency to a lower improvement of $6 \mathrm{MWD}$ as compared to the IPAH/HPAH patients $(85 \pm 49$ versus $56 \pm 49 \mathrm{~m} ; \mathrm{p}=0.086)$. The patients with inoperable CTEPH showed an improvement in 6MWD of $65 \pm 48 \mathrm{~m}$ after 3 weeks $(p<0.001)$ and of $85 \pm 50 \mathrm{~m}$ after 15 weeks $(\mathrm{p}<0.001)$ (table 3 and fig. 2).

The improvement in 6MWD was consistent among different WHO classes (fig. 3). The mean increase in 6MWD compared to baseline in patients in WHO functional class IV was $59 \pm 51 \mathrm{~m}$ after 3 weeks and $63 \pm 61 \mathrm{~m}$ after 15 weeks (table 3, fig. 3). Compared to WHO functional class II or III patients, the per cent increase in walking distance was even more pronounced in the WHO functional class IV patients, although

\section{TABLE 1 Baseline characteristics of the patients}

Data are presented as $n, n(\%)$ or mean $\pm \mathrm{SD}$. WHO: World Health Organization; IPAH: idiopathic pulmonary arterial hypertension; HPAH: heritable pulmonary arterial hypertension; APAH: associated pulmonary arterial hypertension; $\mathrm{PH}$ : pulmonary hypertension; CTEPH: chronic thromboembolic pulmonary hypertension; PCWP: pulmonary capillary wedge pressure.

this difference did not reach statistical significance (table 3). There was a significant improvement in WHO functional class. After 3 weeks exercise training, 22\% of the WHO functional class IV patients improved to functional class III $(p<0.001)$ and $6 \%$ of WHO functional class III to II.

All results remained statistically significant when using the last observation (after 3 weeks) carried forward method. Comparing the patients who completed the study with patients who did not come to the last visit after 15 weeks, dropouts had a higher mean body mass index at baseline and improved significantly but $\sim 10 \%$ less in $6 \mathrm{MWD}$ at 3 weeks than patients who completed the study (69 versus $76 \mathrm{~m}$, respectively; $\mathrm{p}<0.05)$.

Mean peak oxygen consumption, peak oxygen uptake in per cent of the predicted value, and mean peak oxygen pulse of all 


TABLE 2 Pulmonary hypertension (PH)-targeted
medication

Data are presented as $\mathrm{n}(\%) .{ }^{\#}$ : in patients with $\mathrm{PH}$ due to left heart disease or lung disease

patients increased significantly from baseline to 3 weeks and to 15 weeks (table 3). The Borg scale remained unchanged although significantly higher workloads and higher heart rates during exercise were attained (table 3). After 3 weeks of exercise training, mean heart rate, mean diastolic blood pressure and mean systolic pulmonary artery pressure at rest were significantly reduced (table 3 ). Differences in the heart rate after 15 weeks could be influenced by the time point obtaining the ECGs. ECGs after 15 weeks were performed right after the patients had travelled to the hospital, usually at noon or afternoon, ECGs at baseline and after 3 weeks had been performed in the morning in patients who stayed in the hospital. It is possible that the higher stress coming to this examination and the later time within the day led to a higher mean heart rate at the 15 -week visit.

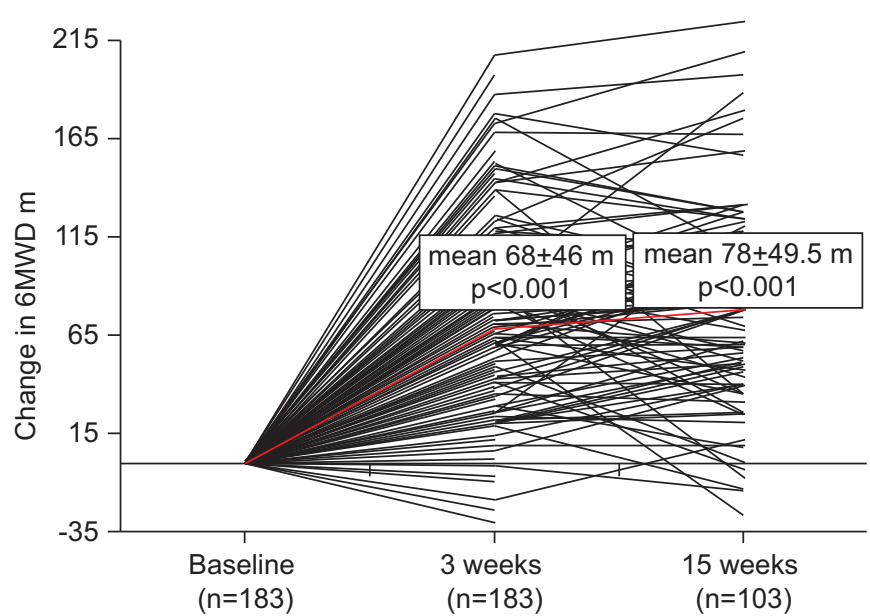

FIGURE 1. Individual changes in 6-min walk distance (6MWD) after 3 and 15 weeks exercise training. With the use of a two-sample t-test stratified according to baseline walking distance, $p<0.001$ was obtained for the comparison to baseline with week 3 and week 15. In this intention-to-treat analysis after 3 weeks of exercise training the data from 183 patients, after 15 weeks from 103 patients, were available and included. The coloured line indicates the mean change from baseline in 6MWD.

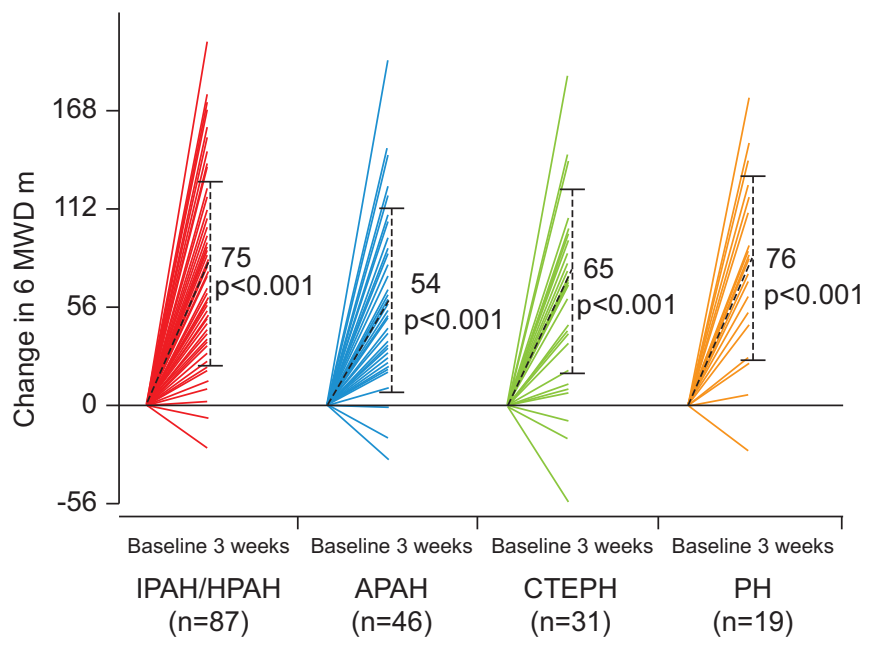

FIGURE 2. Individual changes in the 6-min walk distance (6MWD) in pulmonary hypertension subgroups with different aetiologies from baseline to week 3 and 15. IPAH: idiopathic pulmonary arterial hypertension; HPAH: hereditary pulmonary arterial hypertension; APAH: associated pulmonary arterial hypertension; CTEPH: chronic thromboembolic pulmonary hypertension; $\mathrm{PH}$ : pulmonary hypertension due to respiratory or left heart diseases. The change in 6MWD was not significantly different between the subgroups $(p=0.086)$. The dashed line indicates the mean change in 6MWD from baseline with $95 \%$ confidence intervals.

Furthermore, exercise and respiratory training significantly improved quality of life parameters indicated by the SFsubscale scores for: physical functioning $(p<0.001)$, role physical $(p=0.001)$, role emotional $(p=0.005)$, social functioning $(p=0.037)$, mental health $(p=0.006)$ and vitality ( $p=0.002$; fig. 4 ; table 3 ). Using Bonferroni adjustment, $p$ values $<0.016(6 \mathrm{MWD})$ and $<0.005$ (SF-36) remained statistically significant.

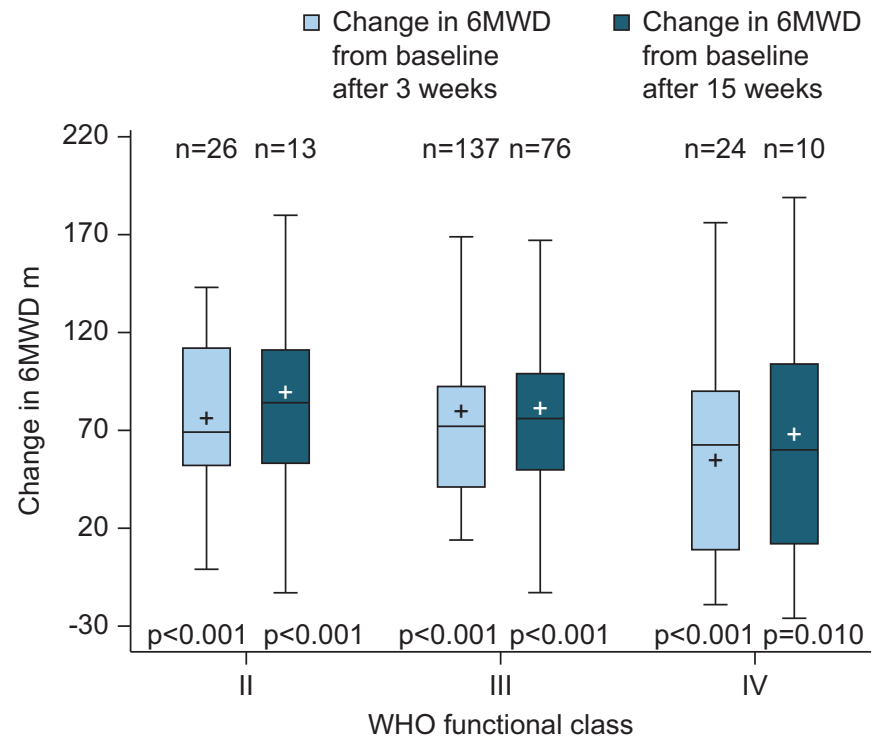

FIGURE 3. Change in 6-min walk distance (6MWD) after 3 and 15 weeks according to World Health Organization (WHO) functional class, indicated as mean $(+)$, median and interquartile range (box), and minimum and maximum values. 
TABLE 3 Efficacy parameters

Characteristics

\begin{tabular}{l} 
Characteristics \\
\hline Subjects $\mathbf{n}$ \\
6MWD by PH aetiology $\mathbf{m}$ \\
All \\
IPAH/HPAH $(n=87 / 87 / 53)$ \\
APAH $(n=46 / 46 / 18)$ \\
CTEPH $(n=31 / 31 / 18)$ \\
PH $(n=19 / 19 / 11)$
\end{tabular}

\section{Difference from baseline 6MWD by PH aetiology $\mathrm{m}$}

All

IPAH/HPAH $(n=87 / 53)$

APAH $(n=46 / 18)$

CTEPH $(n=31 / 18)$

$\mathrm{PH}(\mathrm{n}=19 / 11)$

\section{MWD in WHO-FC subgroups $m$}

WHO-FC II $(n=26 / 26 / 13)$

WHO-FC III $(n=137 / 137 / 76)$

WHO-FC IV $(n=18 / 18 / 10)$

\section{Difference from baseline 6MWD in}

\section{WHO-FC subgroups $m$}

All $(n=183 / 103)$

WHO-FC II $(n=26 / 13)$

WHO-FC III $(n=137 / 76)$

WHO-FC IV $(n=18 / 10)$

\section{Improvement in 6MWD in WHO-FC subgroups \% \\ WHO-FC II $(n=26 / 13)$ \\ WHO-FC III $(n=137 / 76)$ \\ WHO-FC IV $(n=18 / 10)$}

\section{Cardiopulmonary exercise testing}

Peak $V^{\prime} \mathrm{O}_{2}$ per $\mathrm{kg} \mathrm{mL} \cdot \mathrm{min}^{-1} \cdot \mathrm{kg}^{-1}$

Peak $V^{\prime} \mathrm{O}_{2} \mathrm{~mL} \cdot \mathrm{min}^{-1}$

$\mathrm{EqCO}_{2}$ at $\mathrm{AT}$

$V^{\prime} \mathrm{O}_{2}$ at $A T \mathrm{~mL} \cdot \mathrm{min}^{-1}$

Oxygen pulse

PASP rest $\mathrm{mmHg}$

PASP maximum $\mathrm{mmHg}$

Heart rate rest $\mathrm{min}^{-1}$

Heart rate maximum min $^{-1}$

RRsys rest $\mathrm{mmHg}$

RRdia rest $\mathrm{mmHg}$

RRsys maximum $\mathrm{mmHg}$

RRdia maximum $\mathrm{mmHg}$

Borg scale

Workload maximum W

$\frac{\text { Baseline }}{\text { Mean } \pm \text { SD } 95 \% \text { Cl }} \frac{3 \text { weeks }}{\text { Mean } \pm \text { SD Median } 95 \% \text { Cl }}$

183

$\begin{array}{ccc}425 \pm 106 & 410-441 & 493 \pm 110 \\ 448 \pm 89 & 429-467 & 523 \pm 89 \\ 427 \pm 113 & 393-461 & 482 \pm 123 \\ 417 \pm 110 & 377-457 & 482 \pm 126 \\ 327 \pm 100 & 279-375 & 403 \pm 86\end{array}$

183

$\begin{array}{ccc}478-510 & <0.001 & 506 \pm 104 \\ 503-542 & <0.001 & 543 \pm 74 \\ 445-518 & <0.001 & 454 \pm 132 \\ 436-528 & <0.001 & 512 \pm 81 \\ 362-444 & <0.001 & 407 \pm 118\end{array}$

$61-75$

69-92

36-80

52-100

44-118

$<0.001$

$<0.001$

$<0.001$

$<0.001$

$65 \pm 48$

$76 \pm 55$

$\begin{array}{ccc}507 \pm 100 & 466-548 & 572 \pm 100 \\ 424 \pm 86 & 410-439 & 494 \pm 93 \\ 239 \pm 95 & 190-291 & 298 \pm 90\end{array}$

532-613

478-510

253-342

$<0.001$

$<0.001$

$<0.001$

$588 \pm 57$

$511 \pm 85$

$287 \pm 108$
61-75

49-82

62-78

33-84

$<0.001$

$<0.001$

$<0.001$

$<0.001$

$78 \pm 50$
$85 \pm 53$
$79 \pm 48$
$63 \pm 61$

$14.3 \pm 11.1$

$17.5 \pm 13.5$

$25.0 \pm 27.0$

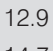

14.7

17.4$$
15
$$
$13.6 \pm 4$
$1006 \pm 308$
$43.6 \pm 10.6$
$712 \pm 236$
$7.9 \pm 2.4$
$57 \pm 20$
$91 \pm 26$
$74 \pm 11$
$127 \pm 22$
$114 \pm 15$
$75 \pm 9$
$151 \pm 24$
$86 \pm 12$
$15.6 \pm 2$
$77 \pm 28$

15 weeks

$95 \% \mathrm{Cl}$ p-value $\begin{array}{cc}486-528 & <0.001 \\ 522-563 & <0.001 \\ 392-516 & <0.001 \\ 473-551 & 0.001 \\ 328-486 & <0.001\end{array}$

$68-88<0.001$

$71-98<0.001$

$32-80<0.001$

60-110 $\quad 0.001$

$41-105<0.001$

554-623 $<0.001$

$492-530<0.001$

$209-364 \quad 0.010$

$68-88<0.001$

$53-117<0.001$

$68-90<0.001$

19-107 $\quad 0.010$

6MWD: 6-min walk distance; IPAH: idiopathic pulmonary arterial hypertension; HPAH: heritable pulmonary arterial hypertension; APAH: associated pulmonary arterial hypertension; CTEPH: chronic thromboembolic pulmonary hypertension; PH: pulmonary hypertension; WHO: World Health Organization; FC: functional class; $\mathrm{V}^{\prime} \mathrm{O}_{2}$ : oxygen uptake; $\mathrm{EqCO}_{2}$ : respiratory equivalence for carbon dioxide; AT: anaerobic threshold; PASP: systolic pulmonary artery pressure; RRsys: systolic systemic blood pressure; RRdia: diastolic systemic blood pressure. p-values are the same for absolute values and differences in 6MWD; Kruskall Wallis test showed no significance in intergroup comparison, $p=0.446$ ( 3 weeks) and $p=0.993$ ( 15 weeks). $p$-values for 6 MWD from two-sided t-test, for cardiopulmonary exercise testing from Wilcoxon test. 


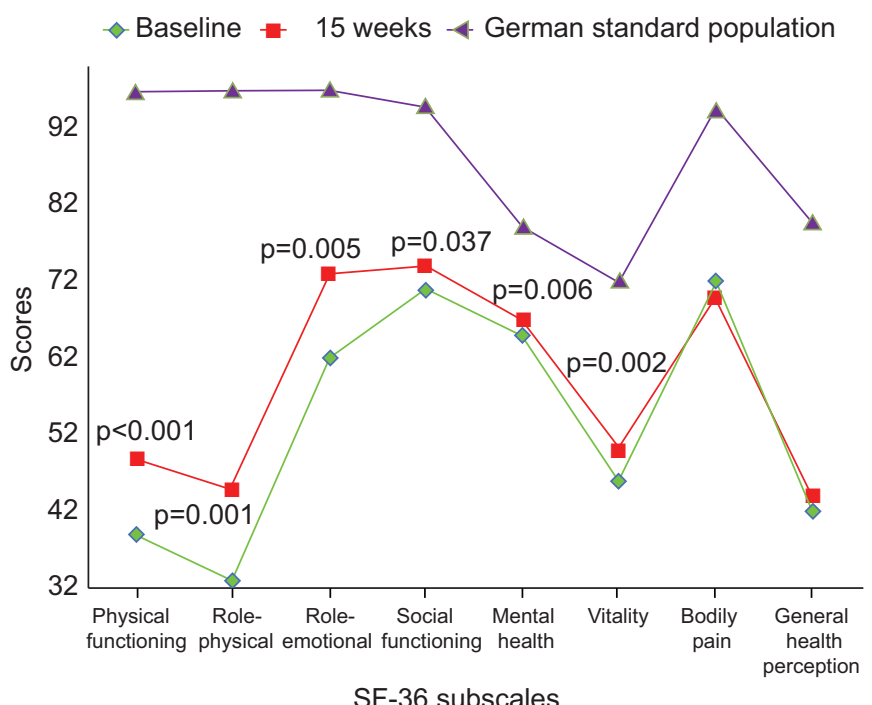

FIGURE 4. Mean Short Form Health Survey (SF-36) scores of quality of life subscales at baseline and after 15 weeks of exercise training. At baseline (green line), mean SF-36 scores were significantly reduced in comparison to respective values of a normal population (lilac line). After 15 weeks (red line), six scales of the SF-36 questionnaire improved significantly: physical functioning, role physical, role emotional, social functioning, mental health and vitality. p-values are indicated versus baseline. No significant improvement was found for bodily pain or general health perception after 15 weeks of exercise training. With Bonferroni adjustment, values of $p<0.05$ preserve statistical significance. At baseline data from 183 patients, and after 15 weeks from 103 patients, were available and included.

\section{Non-responders}

Non-responders have been defined as patients who did not improve $6 \mathrm{MWD}$ after 3 or 15 weeks' exercise training by $>5 \%$ compared to baseline or patients with a decrease in 6MWD. 26 of the 183 patients (14\%) were classified as non-responders. The reasons for non-effective rehabilitation were severe respiratory tract infections $(n=4)$ and orthopaedic problems (swelling of the knee due to knee arthrosis, $n=4$ ) leading to non-adherence to the training programme. In six additional patients, mental disorders, such as depression and anxiety, were diagnosed, and psychopharmacological treatment was required. 11 of the 26 non-responders had no significant improvement in their $6 \mathrm{MWD}$ after 3 weeks, although they participated in the training programme. One patient had performed training before entering the study at home and reported that the training intensity was possibly too low within the 3 weeks in-hospital rehabilitation. Eight of the remaining 10 non-responders had a $6 \mathrm{MWD}$ of $>550 \mathrm{~m}$ at baseline, indicating that the programme is less effective in patients who already have a near to normal 6MWD. Nevertheless, several of these non-responders, who did not improve during the in-hospital stay due to the reasons mentioned above, improved their 6MWD after 15 weeks' exercise training at home. Their increase in quality of life parameters was not significantly different to the "responding" patients indicating, that they did not achieve a significant increase in 6MWD but in quality of life scores. No significant difference between non-responders and the other patients could be found in any parameter listed in table 3. Distribution of WHO functional class, medication and adverse events were similar in the 26 non-responders and the other patients.

\section{Safety during 3-week in-hospital training}

During the 3-week in-hospital training 25 patients (13.6\%) had adverse events. Two patients had a syncope, which occurred not during the exercise training itself but a few hours later. In one patient the syncope occurred when standing up at night after she had developed a severe respiratory infection. In one patient a presyncope occurred immediately after the end of the bicycle ergometer training and was therefore most likely linked to the intensity of the exercise training programme. Five other patients experienced a presyncope not directly associated with the exercise training programme. 14 patients had to discontinue the training programme for 1-2 weeks due to an acute respiratory infection, 12 of them needed antibiotic therapy. Two patients were diagnosed with episodes of supraventricular tachycardia that occurred during the exercise training and were self-limiting. One patient had an episode of slight haemoptysis, which was most likely related to a respiratory infection, but did not occur again following successful antibiotic therapy so she continued the programme. All other patients tolerated the exercise training well. There were no signs of clinical worsening of right heart failure detectable with the non-invasive examinations during the in-hospital programme. All patients reported that they had improved their awareness of their physical abilities and limitations. No further severe adverse events have been occurred between the visit after 3 weeks and the last visit after 15 weeks, where the patients continued with the exercise training at home.

\section{DISCUSSION}

This is the first prospective clinical trial investigating the efficacy and safety of respiratory and exercise training in a large cohort of patients with various aetiologies of chronic $\mathrm{PH}$, such as CTD-APAH, CHD-APAH, PH due to respiratory or left heart diseases and inoperable CTEPH. Furthermore, the study shows that exercise training can improve prognostic relevant parameters even in patients classified as WHO functional class IV. Quality of life, 6MWD, peak oxygen consumption, WHO functional class, exercise capacity, oxygen pulse and other parameters of cardiopulmonary exercise testing, significantly improved after 3 and 15 weeks compared with baseline in all $\mathrm{PH}$ and $\mathrm{WHO}$ functional class subgroups.

During the first 3 weeks of the in-hospital rehabilitation programme, adverse events occurred in $13.6 \%$ of patients, severe events in $4.4 \%$ including syncopes in two and presyncopes in six patients. Thus, the results of this study indicate that exercise training in $\mathrm{PH}$ is an effective, but not a completely harmless add-on therapy and should carefully be supervised and closely monitored.

The study confirms the findings of previous studies in smaller patient cohorts demonstrating a beneficial effect of exercise training in patients with PAH [1-5]. The magnitude of these effects may vary with different physical therapy regimens and with the condition of the patient at the beginning of rehabilitation. The study suggests that the effect of exercise training is somewhat independent of the aetiology of PH. Even in those subgroups of patients usually responding less well to $\mathrm{PH}-$ targeted medication when compared to IPAH, like CTD-APAH 
[14], CHD-APAH [15-17] and inoperable CTEPH [18], exercise training markedly improved $6 \mathrm{MWD}$, oxygen consumption and quality of life. In patients with inoperable CTEPH, exercise training may become a new therapeutic option as add-on to medical therapy if this can be proven in further randomised controlled trials.

\section{Exercise training in $\mathrm{PAH}$ associated with congenital heart defects}

Data on exercise training in CHD-APAH are lacking, except one study of two patients with Eisenmenger's syndrome and two with congenital heart disease that performed low grade exercise training twice a week for 3 months. This study revealed no adverse events, but no objectively measurable improvement [19]. The 36th Bethesda conference recommended patients with congenital heart disease to perform sport only when pulmonary artery pressures are normal [20]. In our study exercise training was safe and effective in these patients although all of them had largely elevated pulmonary artery pressures. Nevertheless, exercise training in patients with CHD-APAH may address special needs and pathophysiological circumstances. Patients with congenital heart disease often tend to overestimate their actual exercise capacity [21], which has been addressed in our programme by offering mental training. Severe progressive cyanosis during exercise in CHD-APAH patients may impair the positive effects of training or even increase the risk for adverse events. Therefore, we tested the possible effect of high-flow oxygen supply in all cyanotic patients.

\section{Exercise training in patients with connective tissue disease} In patients with CTD-APAH the effect of medication therapy on 6MWD was significantly lower than in IPAH patients [14], possibly due to existing comorbidities, such as concomitant musculoskeletal problems, which we addressed in our programme by increased physiotherapy. Therefore, in our study, exercise training in CTD-APAH was as effective as in patients with IPAH or other forms of PH.

\section{Effect of exercise training in patients classified in WHO functional class IV}

Another interesting finding was the high beneficial effect of exercise training in the most severely impaired patients classified in WHO functional class IV. These patients usually have very limited medical options [8] and, even with combination therapy, a severely impaired quality of life and survival rate [22]. Our data suggest that the more patients have been impaired in their physical abilities at baseline, the better the effect of exercise training in improving 6MWD. The incidence of adverse events was not higher, when compared to the patients in WHO functional class II and III. However, these results need to be interpreted with caution, due to the quite small number of patients $(n=18)$ in this group. Further studies may clarify whether exercise training improves time to clinical worsening and survival in patients with $\mathrm{PH}$ in WHO functional class IV.

\section{Adverse events and non-responders}

The overall rate of severe acute adverse events occurred during the hospital stay (two syncopes, six presyncopes) and has been quite low $(4.34 \%)$ and is comparable or even lower than that of patients in rehabilitation programmes for left heart failure [23]. However, this study documented the potential for acute adverse events occurring during the first 3 weeks of in-hospital rehabilitation programme. We cannot exclude that further or more severe adverse events occur when the exercise training is continued at home. Exercise training should definitely not be initiated in a non-supervised and not adequately monitored setting. The patients need to be trained how to perform safe exercise training at home.

The analysis of patients who were not able to improve their $6 \mathrm{MWD}$ during the 3-week in-hospital training $(14.2 \%$ of the 183 patients cohort) did not reveal any differences in the assessed clinical parameters when compared to the $85.8 \%$ of patients who did improve. Most often the mechanism of nonresponse was simply the inability to perform any training within the 3-week in-hospital stay, due to varying reasons. Furthermore, patients with a 6MWD $>550 \mathrm{~m}$ improved less than patients with impaired 6MWD at baseline. Obviously, training is only effective if it is adequately performed and if there is a limited initial physical capacity. Nevertheless, nonresponder patients may have improved by other parameters (such as quality of life and haemodynamic parameters) than in the $6 \mathrm{MWD}$. In a previous study, changes of functional class and haemodynamic indices were more sensitive in detecting treatment effects of PAH-targeted medication [24].

\section{Clinical and pathophysiological mechanism of action}

The precise mechanism through which exercise-based interventions benefit $\mathrm{PH}$ patients remains unclear. Besides improving endothelial dysfunction, muscular blood supply, gas exchange and strength [2, 25], ventilatory efficiency, and ventricular contractility [26] as described in left heart failure [27], exercise training may reduce adrenergic tone and increase vagal tone. After 3 weeks, heart rate at rest, peak oxygen consumption and oxygen pulse all significantly improved in $\mathrm{PH}$ patients of this cohort. Further studies including right heart catheterisation are necessary, e.g. to analyse whether exercise training improves right ventricular contractile reserve. In this study we performed a combined strength and endurance training programme, which has been described as the optimal exercise prescription in left heart failure [28].

\section{Limitations}

Although we believe this to be the largest prospective study analysing the impact of exercise training in patients with pulmonary hypertension, we acknowledge a number of limitations. The study had no randomised control group and was conducted by a single centre. Our data provide a good rationale for future studies of exercise training in $\mathrm{PH}$ patients and assessed this therapy in various aetiologies of $\mathrm{PH}$ for the first time. However, a large controlled randomised, multicentric study should be performed to confirm these results, especially in patients with inoperable CTEPH, CTD-APAH, CHD-APAH and in patients in WHO functional class IV. The effects of exercise training after 15 weeks may be overestimated due to the missing values of $\sim 40 \%$ of patients who did not perform the last follow-up visit and who gained $\sim 10 \%$ less in 6MWD after 3 weeks than the others. Unfortunately, we did not have funding to provide transportation for patients who lived $>400 \mathrm{~km}$ from Heidelberg and, therefore, often 
missed the last visit. Analysis of the data of the participating centres showed that there was no dropout due to side-effects of the training or due to deaths. In addition, we cannot exclude that patients who had a less pronounced training effect may have been less willing to perform the last visit. Furthermore, it is a general issue of rehabilitation programmes that the therapy cannot be performed in a blinded fashion and that a referral bias towards highly motivated patients with a better outcome may occur.

Baseline 6MWD in our cohort of class IV patients was higher than previously reported [29]. We cannot exclude that patients with less exercise limitations have been selected. However, higher values for 6MWD at baseline could, at least in part, be due to the fact that the test was performed in a large sports hall, in which the patients could walk without any time delay by turns or stops, and that some patients of younger age had a comparatively high 6MWD, despite being in WHO IV, as described previously [24].

Further studies are necessary to determine the effects of training programmes on outcome in patients with pulmonary hypertension.

\section{Conclusion}

The results of this study suggest that low-dose exercise and respiratory training as add-on to medical therapy is safe in a closely supervised setting and may improve exercise capacity and quality of life in patients with various forms of $\mathrm{PH}$. Further randomised trials are required to determine whether there is any survival benefit.

\section{SUPPORT STATEMENT}

The study was funded by a grant from the German pulmonary hypertension association: pulmonale hypertonie (ph) e.v. foundation (Rheinstetten, Germany).

\section{STATEMENT OF INTEREST}

None declared.

\section{ACKNOWLEDGEMENTS}

We would like to thank all patients who participated, the self-help group pulmonale hypertonie e.v. (Rheinstetten, Germany,) and especially B. Kopp, for their support. We are deeply grateful for the support of the clinic director K-H. Markmann, all physicians, therapists and physiotherapists of the Rehabilitation Clinic Köenigstuhl (Heidelberg, Germany) who took part in the rehabilitation programmes.

\section{REFERENCES}

1 Mereles D, Ehlken N, Kreuscher S, et al. Exercise and respiratory training improve exercise capacity and quality of life in patients with severe chronic pulmonary hypertension. Circulation 2006; 114: 1482-1489.

2 de Man FS, Handoko ML, Groepenhoff H, et al. Effects of exercise training in patients with idiopathic pulmonary arterial hypertension. Eur Respir J 2009; 34: 669-675.

3 Wilkinson A, Elliot CA, Condliffe R, et al. A randomisedcontrolled trial of a physiotherapist-led rehabilitation programme in patients with pulmonary hypertension. Eur Respir J 2008; 32: Suppl. 52, 166s.

4 Grünig E, Ehlken N, Ghofrani A, et al. Effect of exercise and respiratory training on clinical progression and survival in patients with severe chronic pulmonary hypertension. Respiration 2011; 81: 394-401.

5 Boutet K, Garcia G, Degano B, et al. Results of a 12-week outpatient cardiovascular rehabilitation in patients with idiopathic pulmonary arterial hypertension (iPAH). Eur Respir J 2008; 32: Suppl. 52, 240 s.

6 Handoko ML, de Man FS, Happé CM, et al. Opposite effects of training in rats with stable and progressive pulmonary hypertension. Circulation 2009; 120: 42-49.

7 Barst RJ, McGoon M, Torbicki A, et al. Diagnosis and differential assessment of pulmonary arterial hypertension. J Am Coll Cardiol 2004; 43: 40-47.

8 Task Force for Diagnosis and Treatment of Pulmonary Hypertension of European Society of Cardiology (ESC); European Respiratory Society (ERS); International Society of Heart and Lung Transplantation (ISHLT), Galiè N, Hoeper MM, Humbert $\mathrm{M}$, et al. Guidelines for the diagnosis and treatment of pulmonary hypertension. Eur Respir J 2009; 34: 1219-1263.

9 Guyatt GH, Pugsley SO, Sullivan MJ, et al. Effect of encouragement on walking test performance. Thorax 1984; 39: 818-822.

10 Ware JE, Sherbourne CD. The MOS 36-item Short-Form health survey (SF-36). Med Care 1992; 304: 73-83.

11 Borg GA. Psychophysical bases of perceived exertion. Med Sci Sports Exerc 1982; 14: 377-381.

12 Wasserman K, Hansen JE, Sue DY, et al. Measurements during integrative cardiopulmonary exercise testing. In: Wasserman K, Hansen JE, Sue DY, et al, eds. Principles of Exercise Testing and Interpretation. Philadelphia, Lea and Febiger, 1994; pp. 53-79.

13 Galiè N, Torbicki A, Barst R, et al. Guidelines on diagnosis and treatment of pulmonary arterial hypertension. Eur Heart J 2004; 25: 2243-2278.

14 Chung L, Liu J, Parsons L, et al. Characterization of connective tissue disease-associated pulmonary arterial hypertension from REVEAL: identifying systemic sclerosis as a unique phenotype. Chest 2010; 138: 1383-1394.

15 Adatia I, Kothari SS, Feinstein JA. Pulmonary hypertension associated with congenital heart disease: pulmonary vascular disease: the global perspective. Chest 2010; 137: 52S-61S.

16 Galiè N, Beghetti M, Gatzoulis MA, et al. Bosentan Randomized Trial of Endothelin Antagonist Therapy-5 (BREATHE-5) Investigators. Bosentan therapy in patients with Eisenmenger syndrome: a multicenter, double-blind, randomized, placebo-controlled study. Circulation 2006; 114: 48-54.

17 Haworth SG, Hislop AA. Treatment and survival in children with pulmonary arterial hypertension: the UK Pulmonary Hypertension Service for Children 2001-2006. Heart 2009; 95: 312-317.

18 Humbert M. Pulmonary arterial hypertension and chronic thromboembolic pulmonary hypertension: pathophysiology. Eur Respir Rev 2010; 19: 59-63.

19 Martínez-Quintana E, Miranda-Calderín G, Ugarte-Lopetegui A, et al. Rehabilitation program in adult congenital heart disease patients with pulmonary hypertension. Congenit Heart Dis 2010; 5: 44-50.

20 Graham TP Jr, Driscoll DJ, Gersony WM, et al. Task Force 2: congenital heart disease. J Am Coll Cardiol 2005; 45: 1326-1333.

21 Gratz A, Hess J, Hager A. Self-estimated physical functioning poorly predicts actual exercise capacity in adolescents and adults with congenital heart disease. Eur Heart J 2009; 30: 497-504.

22 Humbert M, Sitbon O, Simmoneau G. Treatment of pulmonary arterial hypertension. $N$ Engl J Med 2004; 351: 1425-36.

23 O'Connor CM, Whellan DJ, Lee KL, et al. Efficacy and safety of exercise training in patients with chronic heart failure: HF-ACTION randomized controlled trial. JAMA 2009; 301: 1439-1450.

24 Degano B, Sitbon O, Savale L, et al. Characterization of pulmonary arterial hypertension patients walking more than $450 \mathrm{~m}$ in $6 \mathrm{~min}$ at diagnosis. Chest 2010; 137: 1297-1303. 
25 Hambrecht R, Niebauer J, Fiehn E, et al. Physical training in patients with stable chronic heart failure: effects on cardiorespiratory fitness and ultrastructural abnormalities of leg muscles. J Am Coll Cardiol 1995; 25: 1239-1249.

26 Belardinelli R, Georgiou D, Ginzton L, et al. Effects of moderate ET on thallium uptake and contractile response to low-dose dobutamine of dysfunctional myocardium in patients with ischemic cardiomyopathy. Circulation 1998; 97: $553-561$.
27 Smart N, Marwick TH. Exercise training for patients with heart failure: a systematic review of factors that improve mortality and morbidity. Am J Med 2004; 116: 693-706.

28 Davies EJ, Moxham T, Rees K, et al. Exercise training for systolic heart failure: Cochrane systematic review and meta-analysis. Eur J Heart Fail 2010; 12: 706-715.

29 Humbert M, Sitbon O, Chaouat A, et al. Pulmonary arterial hypertension in France: results from a national registry. $A m \mathrm{~J}$ Respir Crit Care Med 2006; 173: 1023-1030. 